\title{
Transcriptome-Based Identification of Differently Expressed Genes from Xanthomonas oryzae pv. oryzae Strains Exhibiting Different Virulence in Rice Varieties
}

\author{
Tae-Hwan Noh ${ }^{1,+}$, Eun-Sung Song ${ }^{2,+}$, Hong-Il Kim ${ }^{3,+}$, Mi-Hyung Kang ${ }^{1}$ and Young-Jin Park ${ }^{3, *}$ \\ 1 National Institute of Crop Science, Rural Development Administration, Wanju-gun 55365, Korea; \\ nohtw831@korea.kr (T.-H.N.); kangmh@korea.kr (M.-H.K.) \\ 2 National Institute of Agricultural Science, Rural Development Administration, Jeonju 54874, Korea; \\ ses5494@korea.kr \\ 3 Department of Biomedical Chemistry, Konkuk University, Chungju 27478, Korea; kwangdae7@kku.ac.kr \\ * Correspondence: yjpark@kku.ac.kr; Tel./Fax: +82-43-8403-601 \\ $\dagger$ These authors contributed equally to this work.
}

Academic Editors: Patrick C. Y. Woo and Dilantha Fernando

Received: 4 January 2016; Accepted: 16 February 2016; Published: 19 February 2016

\begin{abstract}
Xanthomonas oryzae pv. oryzae (Xoo) causes bacterial blight (BB) in rice (Oryza sativa L.). In this study, we investigated the genome-wide transcription patterns of two Xoo strains (KACC10331 and HB1009), which showed different virulence patterns against eight rice cultivars, including IRBB21 (carrying Xa21). In total, 743 genes showed a significant change ( $p$-value $<0.001$ in $t$-tests) in their mRNA expression levels in the HB1009 (K3a race) strain compared with the Xoo KACC10331 strain (K1 race). Among them, four remarkably enriched GO terms, DNA binding, transposition, cellular nitrogen compound metabolic process, and cellular macromolecule metabolic process, were identified in the upregulated genes. In addition, the expression of 44 genes was considerably higher ( $\log 2$ fold changes > 2) in the HB1009 (K3a race) strain than in the Xoo KACC10331 (K1 race) strain. Furthermore, 13 and 12 genes involved in hypersensitive response and pathogenicity ( $h r p)$ and two-component regulatory systems (TCSs), respectively, were upregulated in the HB1009 (K3a race) strain compared with the Xoo KACC10331 (K1 race) strain, which we determined using either quantitative real-time PCR analysis or next-generation RNA sequencing. These results will be helpful to improve our understanding of Xoo and to gain a better insight into the Xoo-rice interactions.
\end{abstract}

Keywords: bacterial blight; differently expressed gene; transcriptome; virulence; Xanthomonas oryzae pv. oryzae

\section{Introduction}

Bacterial blight (BB) has become one of the most devastating diseases of rice and an important problem affecting rice production worldwide. Some bactericides have been developed to control the disease, but none is highly effective. Thus, genetic basis breeding for BB resistance has been found to be the most effective method for the control of BB [1]. At least $38 \mathrm{BB}$ resistance $(R)$ genes in rice have been identified [2,3], of which six (Xa2, Xa4, Xa7, Xa30, Xa33, and Xa38) have been physically mapped and eight (Xa1, xa5, xa13, Xa21, Xa26/Xa3, Xa10, Xa23 and Xa27) have been cloned [2-11]. Among them, the Xa21 gene was identified in the wild species Oryza longistaminata and shown to be highly effective against BB races of South and Southeast Asia [12]. Pattern recognition receptors (PRRs) are proteins of critical importance in detecting invading pathogens in plants and animals. 
In rice, $X A 21$ is a well-characterized PRR and is a leucine-rich repeat receptor-like kinase that confers broad-spectrum resistance to multiple strains of the bacterial pathogen Xoo. Several bacterial genes that are required for activation of XA21-mediated immunity ( $r a x$ genes) have been identified [13-15]. Studies by Lee $e t$ al. [16] on XA21-mediated immunity activation showed that a mutation in the Xoo protein (PXO_03968), a 198-aa-long protein named Ax21 (for Activator of XA21-mediated immunity), allowed the bacterium to evade XA21-mediated immunity. However, further analysis of this gene revealed that a mutation in ax21 (PXO_03968) still activated XA21-mediated immunity [17].

Korean Xoo isolates have been classified into five races (K1 to K5) based on the Xoo differential system with five rice cultivars [18]. Recent pathotype analysis of the Korean Xoo isolates revealed that the $\mathrm{K} 1$ race has decreased, whereas the $\mathrm{K} 2$ and $\mathrm{K} 3$ races have increased in Korea [19-21]. In addition, Noh et al. [22] reported that a new Xoo race (K3a) is pathogenic for rice cultivars, possesses the Xa1, $\mathrm{X} a 3$, or $\mathrm{X} a 4$ gene according to a virulence assay, and caused serious damage to rice production in the southwestern coastal areas of Korea in 2003 [22]. Recently, Song et al. [23] reported that the AFLP-derived marker could be used for the specific detection of the K3a race. This marker is important because early identification of this race by rapid methods is important for assessing the health status of rice. However, fundamental studies on the properties of this new race remain challenging.

These two aspects, i.e., that Ax21 is not the activator of XA21-mediated immunity and the reports regarding the new Xoo race (K3a) outbreak, motivated us to carry out more detailed studies based on genome-wide transcription analysis with Xoo KACC10331 (virulent against IRBB21) and Xoo HB1009 (K3a race). Here, we report the genome-wide transcription profiling of genes in two Xoo strains, one ( $\mathrm{K} 1$ race) is virulent against IRBB21 (carrying Xa21) and the other is a new Xoo race (K3a) that is pathogenic for rice cultivars and possesses the Xa1, Xa3, or Xa4 gene.

\section{Results}

\subsection{Virulence of the Xoo K1 Race and K3a Race Strains}

In a virulence assay, one susceptible rice cultivar (IR24) and seven near-isogenic lines (NILs) against bacterial blight, including IRBB1 (carrying Xa-1), IRBB2 (carrying Xa-2), IRBB3 (carrying Xa-3), IRBB4 (carrying Xa-4), IRBB5 (carrying Xa-5), IRBB7 (carrying Xa-7), and IRBB21 (carrying Xa-21), from the International Rice Research Institute (IRRI), were inoculated with the Xoo KACC10331 (K1 race) and HB1009 (K3a race) strains. There were no significant differences between the lesion lengths of three varieties (IR24, IRBB4, and IRBB5) when inoculated with the two strains. However, the two strains showed different virulence patterns against the rice varieties IRBB1, IRBB2, IRBB3, IRBB7, and IRBB21. Twenty-one days after inoculation, the average lengths of the lesions caused by the KACC10331 (K1 race) strain were $1 \pm 0,0.5 \pm 0,3.7 \pm 0.6$, and $1.2 \pm 0.3 \mathrm{~cm}$ against IRBB1, IRBB2, IRBB3, and IRBB7, respectively, whereas those of the lesions caused by the HB1009 (K3a race) strain were $15.6 \pm 2,14.6 \pm 0.5,11.5 \pm 0.5$, and $7.0 \pm 0 \mathrm{~cm}$, respectively (Figure 1). In contrast, for IRBB21, the average lesion length caused by the KACC10331 (K1 race) strain was $15.7 \pm 2.0 \mathrm{~cm}$, whereas that of the lesion caused by the HB1009 (K3a race) strain was $4.3 \pm 0.5 \mathrm{~cm}$ (Figure 1).

Our results indicated that the virulence of the KACC10331 (K1 race) strain was significantly weaker than that of the HB1009 (K3a race) strain when infecting four rice varieties (IRBB1, IRBB2, IRBB3, and IRBB7). However, the virulence of the KACC10331 (K1 race) strain was significantly stronger than that of the HB1009 (K3a race) strain when infecting IRBB21 (carrying the Xa-21 resistance gene) NIL. 
A

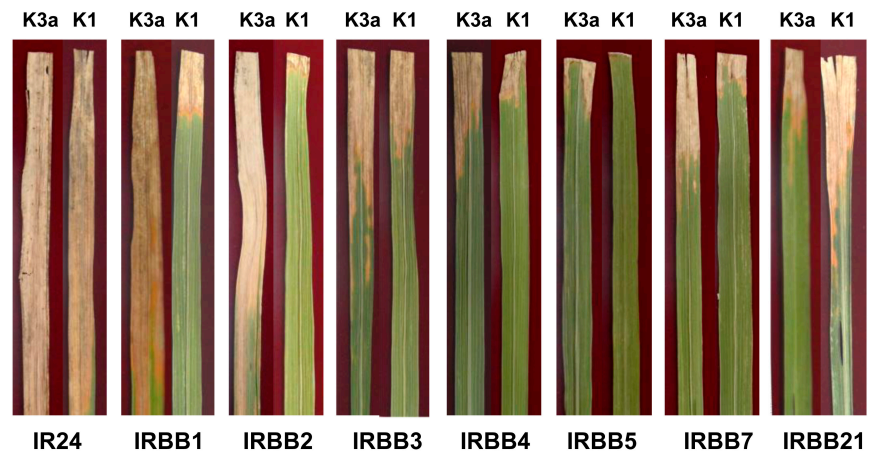

B

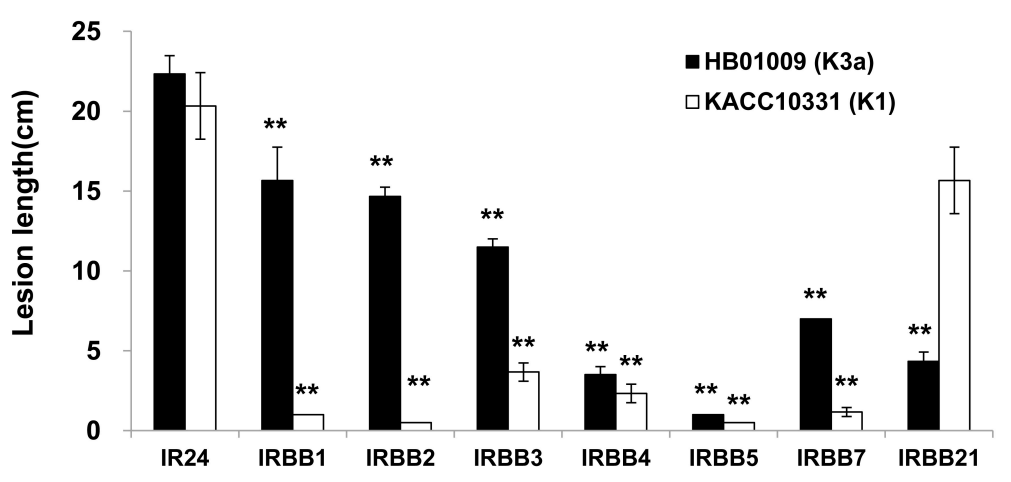

Figure 1. Virulence assay (A) and lesion length (B) of rice varieties inoculated with two Xanthomonas oryzae pv. oryzae strains (K1 and K3a race). Asterisks represent statistically significant differences relative to a susceptible rice cultivar (IR24) (paired, two-tailed Student's $t$ test, ** $p$-value $<0.01)$.

\subsection{RNA-seq Reads Mapping and GO Term Enrichment of Differentially Expressed Genes}

To compare the transcriptome profiles of the Xoo KACC10331 (K1 race) and HB1009 (K3a race) strains, RNA sequencing libraries were constructed. The two libraries generated about 16.1 and 19.6 million reads, which were mapped to the X. oryzae pv. oryzae KACC10331 genome sequence in the NCBI GenBank database (NC_006834.1) with 96.9\% and 96.4\% matched reads to NCBI annotated gene regions (Figure 2). There were 4065 protein-coding genes (CDS) among 4281 annotated genes in the genome of KACC10331 by genome analysis [24]. A total of 4062 and 3995 protein-coding genes were detected ( $\geqslant 5$ mapped reads) in the Xoo KACC10331 (K1 race) and HB1009 (K3a race) strains, respectively (Figure 2).

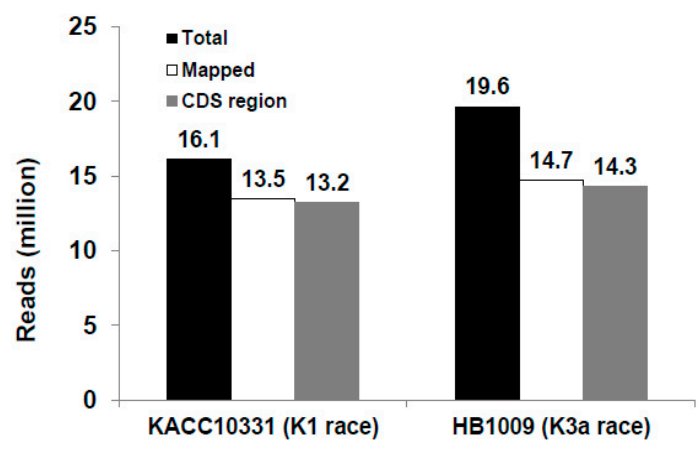

Figure 2. Total number of R-seq reads from each Xanthomonas oryzae pv. oryzae strain library and mapped reads to the Xanthomonas oryzae pv. oryzae KACC10331 genome. 
To identify DEGs, we used the R-package DEGseq [25]. Genes with significant differences in expression level between the two strains were evaluated using the criterion of a $p$-value less than 0.001 in a $t$-test, resulting in 743 DEGs when the HB1009 (K3a race) strain was compared with the Xoo KACC10331 strain (K1 race) (Table S1). To further analyze these DEGs, the transcripts were categorized according to their annotated function with respect to biological processes, molecular functions, and cellular components, on the basis of the blast and GO term annotation using Blast2GO software [26]. The biological processes mediated by these DEGs were primarily associated with cellular processes, metabolic processes, and single organism processes, among others (Figure 3, Table S2). The most major molecular functions were binding and catalytic activity. Cell was the most majority in the cellular component category. Most of these categories contained a larger numbers of genes with typically upregulated expression than those of genes with typically downregulated expression (Figure 3, Table S2). In the GO term enrichment analysis using Blast2GO (Fisher's exact test), four remarkably enriched GO terms, DNA binding, transposition, cellular nitrogen compound metabolic process, and cellular macromolecule metabolic process, were identified in the upregulated genes. However, there were no GO terms enriched in the genes with downregulated expression (Table S3). Thus, the RNA-seq and GO term enrichment analysis results suggested that some of these genes were predominantly expressed in HB1009 (K3a race), since the expression of 44 genes (a total of 147 enriched genes) was considerably higher (log2 fold changes > 2) in the HB1009 (K3a race) strain than in the Xoo KACC10331 (K1 race) strain (Table S4).

\section{$\square$ upregulated $\square$ downregulated}

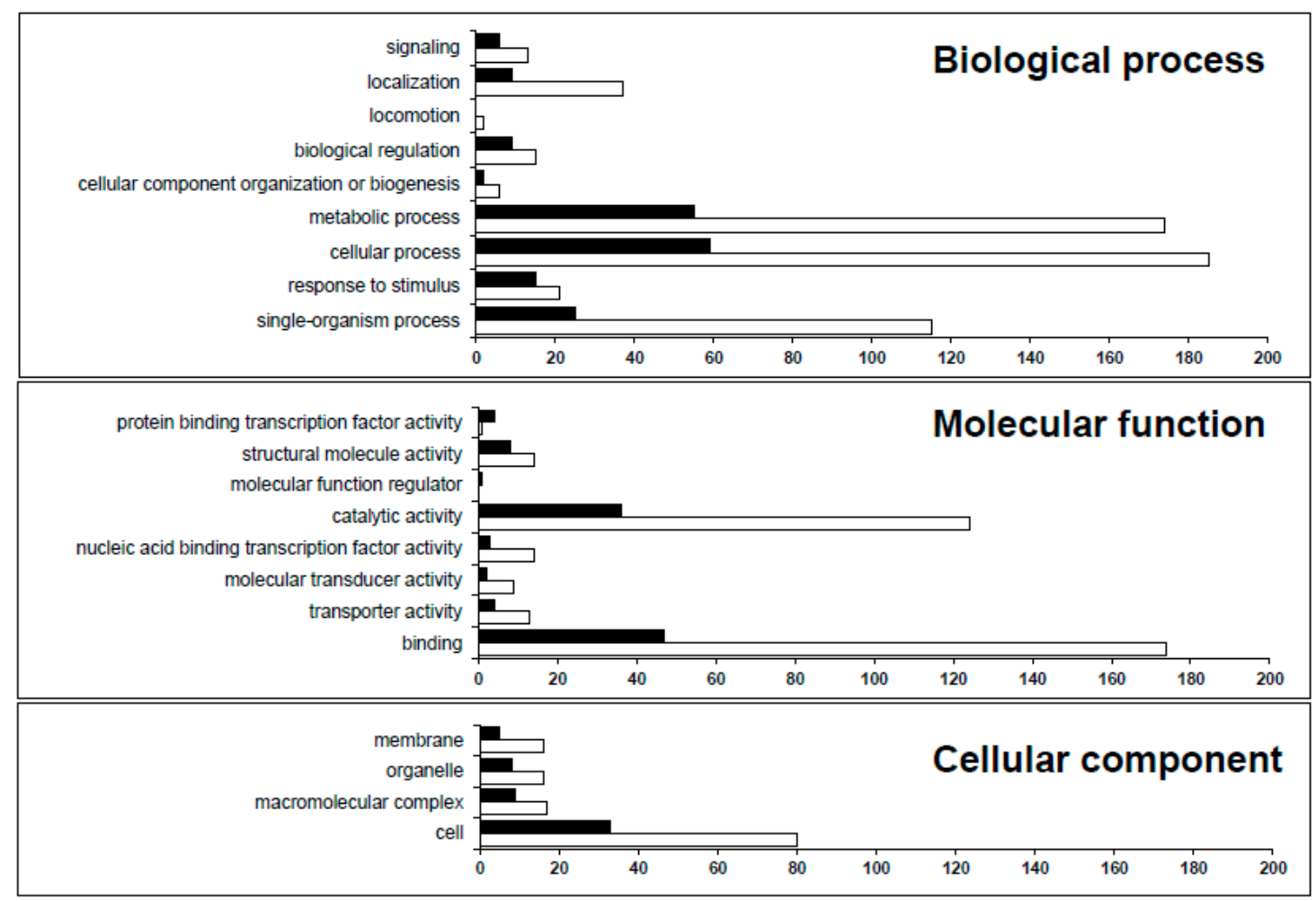

Figure 3. Functional categorization of the upregulated and downregulated genes in Xanthomonas oryzae pv. oryzae HB1009 (K3a race) based on the GO annotation.

\subsection{Differential Expression of hrp Genes between the Xoo K1 Race and K3a Race Strains}

In xanthomonads, the hrp (hypersensitive response and pathogenicity) genes are highly conserved and clustered, which is essential for the pathogenicity of Xoo [27,28]. According to the genome sequence of Xoo KACC10331, the hrp gene cluster was composed of nine $h r p$ ( $h r p F, h r p E$, $h r p D 6, h r p D 5$, hrpB1, 
hrpB2, hrpB4, hrpB5, and $h r p B 7)$, nine $h r c$ ( $h r p$ conserved; $h r c S, h r c R, h r c Q, h r c V, h r c U, h r c J(h r p B 3), h r c N$, hrcT (hrpB8), and $h r c C)$, and eight hpa (hrp-associated; hpaF, hpaB, hpaA, hpaP, hpa1, hpa2, hpa3, and hpa4) genes [24,27]. The expression of the $h r p A$ and $h r p X$ genes is thought to be controlled by HrpG, which is the OmpR family regulator [29]. In addition, $h r p X$, which belongs to the AraC family of positive transcriptional activators, controls the expression of operons $h r p B$ to $h r p F$ and a number of virulence factors [30]. In transcriptome analysis, $13 \mathrm{hrp} \mathrm{(hpa4;XOO0074,} \mathrm{hpaB;}$ XOO0075, $h r p D 5 ; \mathbf{X O O 0 0 7 8 , ~ h p a A ;}$ XOO0079, $h r c S ; X O O 0080, h r c R ; X O O 0081, h r c Q ; X O O 0082, h p a P ; \mathbf{X O O 0 0 8 3 , ~ h r p B 2 ; ~ X O O 0 0 8 7 , ~} h r p B 8$; $\mathrm{XOO} 0093$, hpaD6; $\mathrm{XOO} 0077$, hpa2; $\mathrm{XOO} 0096$, and $h p a 3 ; \mathrm{XOO} 4700)$ were significantly upregulated in the HB1009 (K3a race) strain compared with the Xoo KACC10331 (K1 race) strain (Table 1, Figure 4). The log2 FCs of the 13 genes ranged from 0.97 to 2.47. Among them, the expression levels of four hrp genes, including $h p a B$ (XOO0075), hpaA (XOO0079), hrcS (XOO0080), and $h r p B 2$ (XOO0087), were considerably higher ( $\log 2$ fold changes $>2$ ) in the HB1009 (K3a race) strain than in the Xoo KACC10331 (K1 race) strain (Table 1, Figure 4). Most of these upregulated genes involved in the hrp cluster are known to be crucial for the HR and virulence in Xoo [27]. In addition, Zhang et al. [31] reported that an increase in transcript alterations of hrp genes and type III effectors results in increased Xoo virulence. These results suggest that the increased virulence of the HB1009 strain compared with the KACC10331 strain when infecting some rice varieties was due to the differentially upregulated expression of $h r p$ genes.

Table 1. Expression profiles of hrp genes in the Xanthomonas oryzae pv. oryzae KACC10331 and HB1009 strains. * True indicates significant differential expression $(p$-value $<0.001)$.

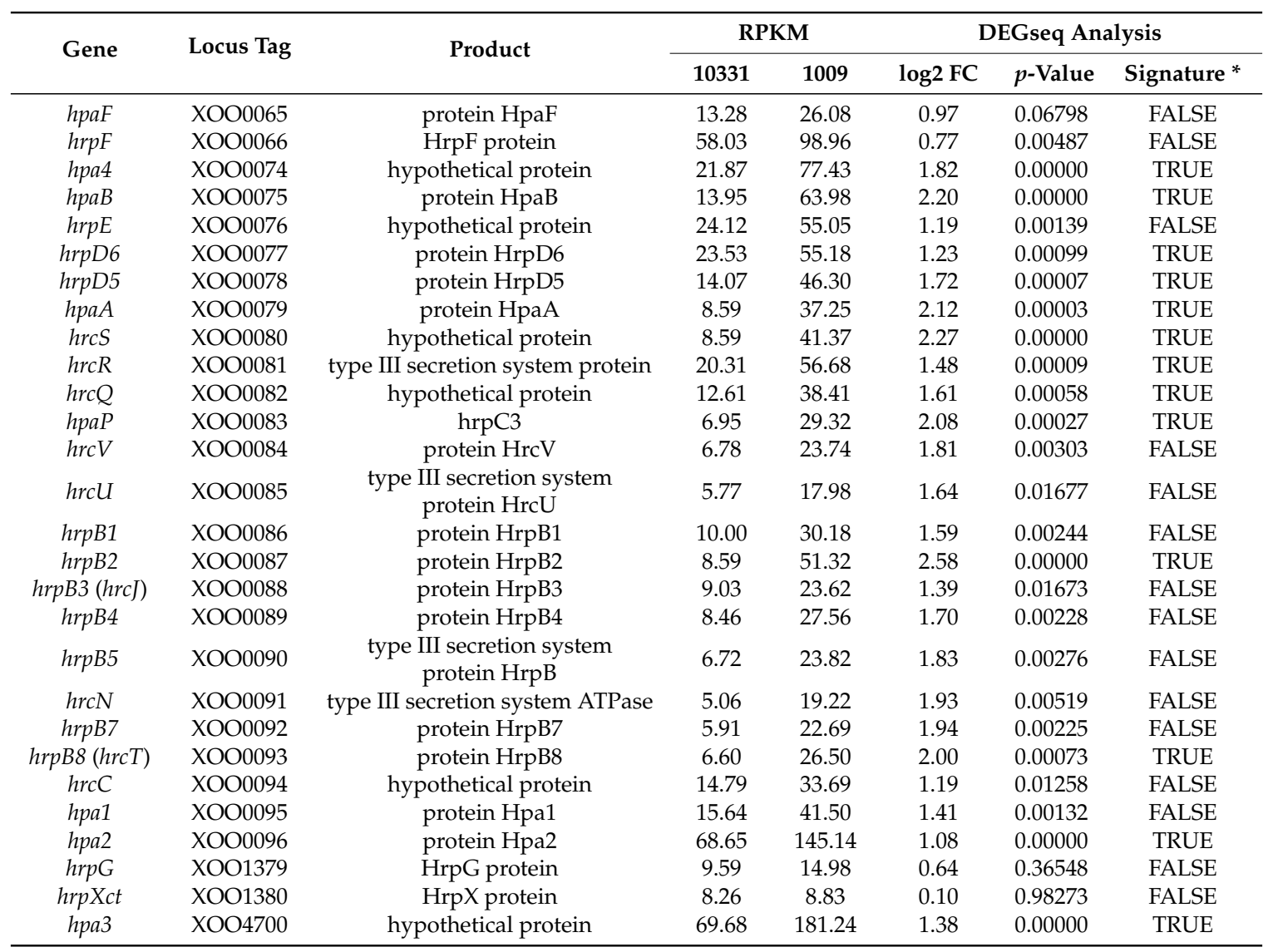


A

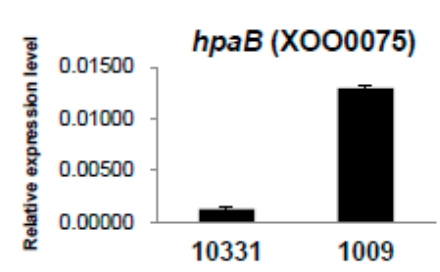

D

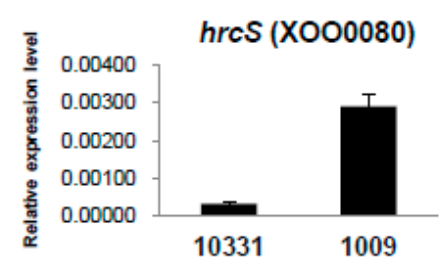

G

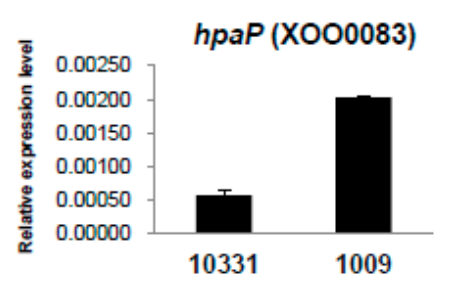

J

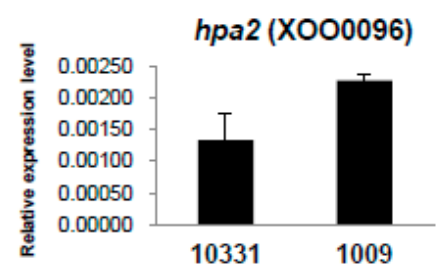

B

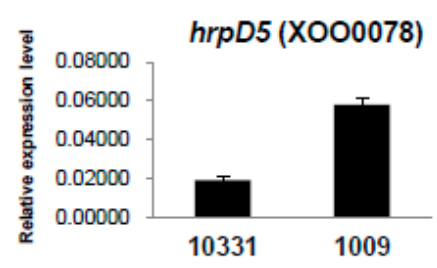

E

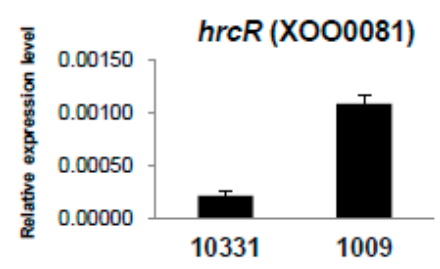

H

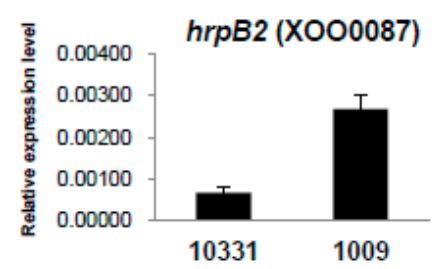

C

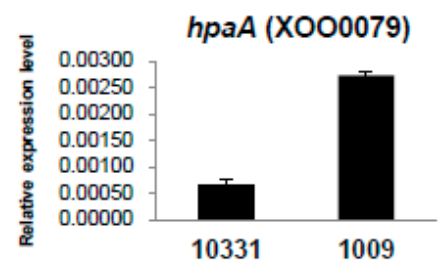

F

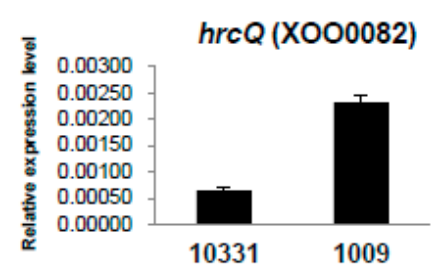

I

Figure 4. The expression patterns of hrp genes of the Xanthomonas oryzae pv. oryzae KACC10331 (K1 race) and HB1009 (K3a race) strains. The gene expression levels (arbitrary units) of $h p a B$ (A); $h r p D 5$ (B); $h p a A(\mathbf{C}) ; \operatorname{hrcS}(\mathbf{D}) ; \operatorname{hrcR}(\mathbf{E}) ; \operatorname{hrcQ}(\mathbf{F}) ; \operatorname{hpaP}(\mathbf{G}) ; \operatorname{hrpB2}(\mathbf{H}) ; \operatorname{hrpB8}(\mathbf{I})$; and $h p a 2(\mathbf{J})$ were normalized using $16 \mathrm{~S}$ RNA as an internal reference. Gene expression levels were quantified by real-time RT-PCR.

\subsection{Differential Expression of Two-Component Systems between the Xoo K1 Race and K3a Race Strains}

To cope with various environmental conditions, including limited-nutrient niches and various toxic substances produced by the host, bacteria have evolved a mechanism involving two-component regulatory systems (TCSs). TCSs are composed of histidine kinases (HKs) and response regulators (RRs). In response to environmental stimuli, the HK phosphorylates the cognate RR, which then regulates gene expression [32]. In this study, among the 48 TCSs, 15 TCSs showed significantly different expression levels in the HB1009 (K3a race) strain compared with Xoo KACC10331 (K1 race). Twelve TCSs (XOO0336, XOO0519, XOO1105, XOO1207, XOO2227, XOO2228, XOO3659, XOO3709, XOO3710, XOO3871, XOO3875, and XOO4008) and three TCSs (XOO0423, XOO2322, and XOO2798) were upregulated and downregulated, respectively, in the HB1009 (K3a race) strain compared with the Xoo KACC10331 (K1 race) strain (Figure 5, Table 2, Table S1). In addition, three (XOO1105, XOO2227, and XOO4008) out of 12 significantly upregulated TCSs were over-represented in the GO term enrichment analysis (Table S4). 
A

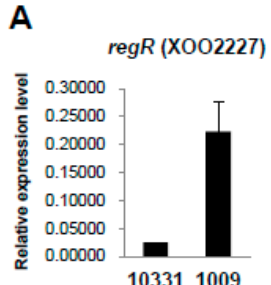

B

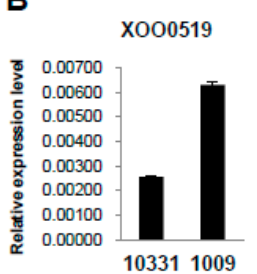

$\mathbf{F} \quad \operatorname{raxP}(\mathrm{X003397)}$
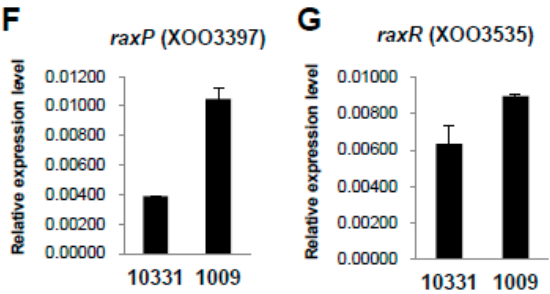
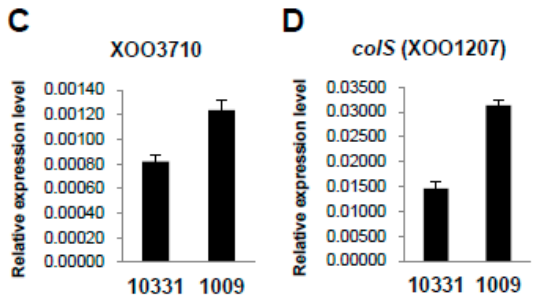

E
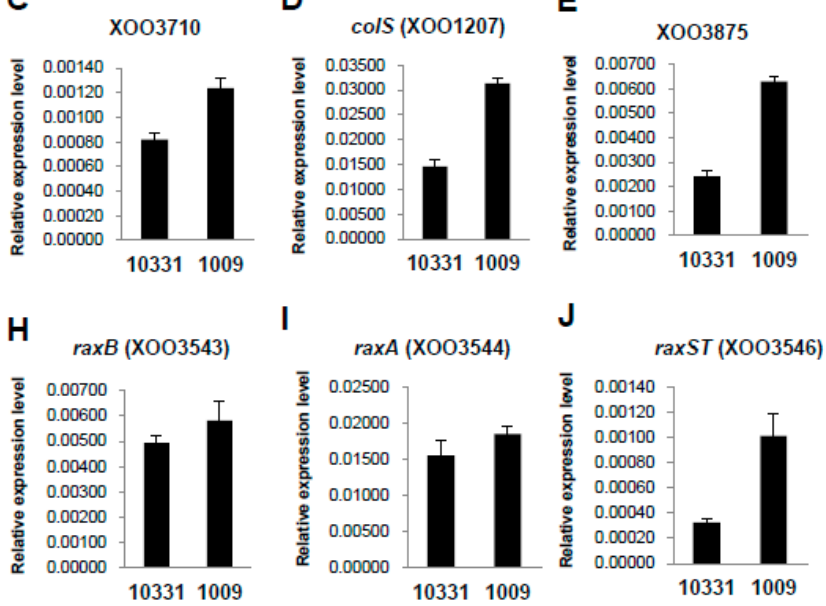

Figure 5. The expression patterns of TCS and rax genes of the Xanthomonas oryzae pv. oryzae KACC10331 (K1 race) and HB1009 (K3a race) strains. The gene expression levels (arbitrary units) of regR (A); XOO0519 (B); XOO3710 (C); colS (D); XOO3875 (E); $\operatorname{raxP}(\mathbf{F}) ; \operatorname{raxR}(\mathbf{G}) ; \operatorname{raxB}(\mathbf{H}) ; \operatorname{raxA}(\mathbf{I})$; and $\operatorname{raxST}(\mathbf{J})$ were normalized using $16 \mathrm{~S}$ RNA as an internal reference. Gene expression levels were quantified by real-time RT-PCR.

Table 2. Expression profiles of TCSs and rax gens in the Xanthomonas oryzae pv. oryzae KACC10331 and HB1009 strains. ${ }^{*}$ True indicates significant differential expression $(p$-value $<0.001)$.

\begin{tabular}{|c|c|c|c|c|c|c|c|}
\hline \multirow{2}{*}{ Gene } & \multirow{2}{*}{ Locus Tag } & \multirow{2}{*}{ Product } & \multicolumn{2}{|c|}{ RPKM } & \multicolumn{3}{|c|}{ DEGseq Analysis } \\
\hline & & & 10331 & 1009 & $\log 2 \mathrm{FC}$ & $p$-Value & Signature * \\
\hline ygiY & XOO0057 & $\begin{array}{l}\text { two-component system } \\
\text { sensor protein }\end{array}$ & 23.33 & 43.05 & 0.77 & 0.03277 & FALSE \\
\hline- & XOO0336 & $\begin{array}{l}\text { two-component system } \\
\text { sensor protein }\end{array}$ & 6.14 & 25.69 & 1.96 & 0.00068 & TRUE \\
\hline phoP & XOO0423 & $\begin{array}{l}\text { two-component system } \\
\text { regulatory protein }\end{array}$ & 143.04 & 99.05 & -0.64 & 0.00061 & TRUE \\
\hline phoQ & XOO0424 & $\begin{array}{l}\text { two-component system } \\
\text { sensor protein }\end{array}$ & 42.90 & 38.67 & -0.26 & 0.41585 & FALSE \\
\hline- & XOO0519 & $\begin{array}{l}\text { two-component system } \\
\text { sensor protein }\end{array}$ & 8.56 & 37.48 & 2.02 & 0.00003 & TRUE \\
\hline- & XOO0520 & $\begin{array}{l}\text { two-component system } \\
\text { regulatory protein }\end{array}$ & 10.45 & 27.08 & 1.26 & 0.01107 & FALSE \\
\hline- & XOO0683 & $\begin{array}{l}\text { two-component response } \\
\text { regulator }\end{array}$ & 14.10 & 16.94 & 0.15 & 0.76601 & FALSE \\
\hline$t c t D$ & XOO1105 & $\begin{array}{l}\text { two-component system } \\
\text { regulatory protein }\end{array}$ & 58.18 & 117.85 & 0.91 & 0.00005 & TRUE \\
\hline$t c t E$ & X001106 & $\begin{array}{l}\text { two-component system } \\
\text { sensor protein }\end{array}$ & 21.90 & 52.13 & 1.14 & 0.00115 & FALSE \\
\hline cols & XOO1207 & $\begin{array}{l}\text { two-component system } \\
\text { sensor protein }\end{array}$ & 66.31 & 231.53 & 1.69 & 0.00000 & TRUE \\
\hline colR & XOO1208 & $\begin{array}{l}\text { two-component system } \\
\text { regulatory protein }\end{array}$ & 26.59 & 48.57 & 0.76 & 0.02578 & FALSE \\
\hline $\mathrm{creC}$ & XOO1477 & $\begin{array}{l}\text { two-component system } \\
\text { sensor protein }\end{array}$ & 69.24 & 97.83 & 0.39 & 0.08422 & FALSE \\
\hline baes & XOO1558 & $\begin{array}{l}\text { two-component system } \\
\text { sensor protein }\end{array}$ & 3.69 & 13.48 & 1.76 & 0.02205 & FALSE \\
\hline$b a e R$ & XOO1559 & $\begin{array}{l}\text { two-component system } \\
\text { regulatory protein }\end{array}$ & 5.03 & 12.25 & 1.17 & 0.10729 & FALSE \\
\hline pilR & XOO1591 & $\begin{array}{l}\text { two-component system } \\
\text { regulatory protein }\end{array}$ & 21.08 & 18.95 & -0.26 & 0.56344 & FALSE \\
\hline
\end{tabular}


Table 2. Cont.

\begin{tabular}{|c|c|c|c|c|c|c|c|}
\hline \multirow{2}{*}{ Gene } & \multirow{2}{*}{ Locus Tag } & \multirow{2}{*}{ Product } & \multicolumn{2}{|c|}{ RPKM } & \multicolumn{3}{|c|}{ DEGseq Analysis } \\
\hline & & & 10331 & 1009 & $\log 2 \mathrm{FC}$ & $p$-Value & Signature * \\
\hline pils & XOO1592 & $\begin{array}{l}\text { two-component system } \\
\text { sensor protein }\end{array}$ & 5.36 & 10.62 & 0.88 & 0.23802 & FALSE \\
\hline$r e g R$ & XOO2227 & $\begin{array}{l}\text { two-component system } \\
\text { regulatory protein }\end{array}$ & 117.22 & 376.62 & 1.57 & 0.00000 & TRUE \\
\hline regs & XOO2228 & $\begin{array}{l}\text { two-component system } \\
\text { sensor protein }\end{array}$ & 43.39 & 165.10 & 1.82 & 0.00000 & TRUE \\
\hline- & XOO2322 & $\begin{array}{l}\text { two-component system } \\
\text { regulatory protein }\end{array}$ & 124.34 & 48.71 & -1.46 & 0.00000 & TRUE \\
\hline- & XOO2323 & $\begin{array}{l}\text { two-component system } \\
\text { sensor protein }\end{array}$ & 16.32 & 17.80 & 0.02 & 0.97542 & FALSE \\
\hline $\operatorname{rrpX}$ & XOO2787 & transcriptional regulator & 13.09 & 21.30 & 0.59 & 0.23576 & FALSE \\
\hline- & XOO2797 & $\begin{array}{l}\text { two-component system } \\
\text { sensor protein }\end{array}$ & 10.54 & 12.89 & 0.18 & 0.76266 & FALSE \\
\hline- & XOO2798 & $\begin{array}{l}\text { two-component system } \\
\text { regulatory protein }\end{array}$ & 32.57 & 7.65 & -2.20 & 0.00002 & TRUE \\
\hline $\operatorname{rpfC}$ & XOO2870 & RpfC protein & 18.06 & 27.96 & 0.52 & 0.22665 & FALSE \\
\hline$r p f G$ & XOO2871 & response regulator & 39.38 & 52.65 & 0.31 & 0.30732 & FALSE \\
\hline- & XOO3527 & $\begin{array}{l}\text { two-component system } \\
\text { regulatory protein }\end{array}$ & 21.45 & 24.84 & 0.10 & 0.81159 & FALSE \\
\hline- & XOO3528 & $\begin{array}{l}\text { two-component system } \\
\text { sensor protein }\end{array}$ & 9.75 & 14.03 & 0.41 & 0.48663 & FALSE \\
\hline- & XOO3659 & $\begin{array}{l}\text { two-component system } \\
\text { regulatory protein }\end{array}$ & 114.21 & 209.67 & 0.77 & 0.00000 & TRUE \\
\hline phoB & XOO3666 & $\begin{array}{l}\text { two-component system } \\
\text { regulatory protein }\end{array}$ & 11.54 & 18.72 & 0.59 & 0.26937 & FALSE \\
\hline phoR & XOO3667 & $\begin{array}{l}\text { two-component system } \\
\text { sensor protein }\end{array}$ & 4.77 & 13.97 & 1.44 & 0.04384 & FALSE \\
\hline tor $S$ & XOO3709 & $\begin{array}{l}\text { two-component system } \\
\text { sensor protein }\end{array}$ & 7.90 & 34.31 & 2.01 & 0.00006 & TRUE \\
\hline- & XOO3710 & $\begin{array}{l}\text { two-component system } \\
\text { regulatory protein }\end{array}$ & 7.34 & 38.26 & 2.27 & 0.00001 & TRUE \\
\hline cols & XOO3762 & $\begin{array}{l}\text { two-component system } \\
\text { sensor protein }\end{array}$ & 8.93 & 13.62 & 0.50 & 0.41805 & FALSE \\
\hline colR & XOO3763 & $\begin{array}{l}\text { two-component system } \\
\text { regulatory protein }\end{array}$ & 59.32 & 42.52 & -0.59 & 0.04006 & FALSE \\
\hline$k d p E$ & XOO3842 & $\begin{array}{l}\text { two-component system } \\
\text { regulatory protein }\end{array}$ & 5.43 & 13.43 & 1.20 & 0.08655 & FALSE \\
\hline$k d p D$ & XOO3843 & $\begin{array}{l}\text { two-component system } \\
\text { sensor protein }\end{array}$ & 10.16 & 13.90 & 0.34 & 0.56471 & FALSE \\
\hline- & XOO3870 & $\begin{array}{l}\text { two-component system } \\
\text { regulatory protein }\end{array}$ & 35.44 & 60.64 & 0.66 & 0.02655 & FALSE \\
\hline- & XOO3871 & $\begin{array}{l}\text { two-component system } \\
\text { sensor protein }\end{array}$ & 14.79 & 43.80 & 1.46 & 0.00032 & TRUE \\
\hline- & XOO3875 & $\begin{array}{l}\text { two-component system } \\
\text { sensor protein }\end{array}$ & 164.50 & 381.45 & 1.10 & 0.00000 & TRUE \\
\hline- & XOO3935 & $\begin{array}{l}\text { two-component system } \\
\text { regulatory protein }\end{array}$ & 11.04 & 15.52 & 0.38 & 0.49899 & FALSE \\
\hline- & XOO3936 & $\begin{array}{l}\text { two-component system } \\
\text { sensor protein }\end{array}$ & 16.87 & 19.88 & 0.13 & 0.79059 & FALSE \\
\hline $\operatorname{alg} R$ & XOO4008 & $\begin{array}{l}\text { two-component system } \\
\text { regulatory protein }\end{array}$ & 26.39 & 59.48 & 1.06 & 0.00105 & FALSE \\
\hline $\operatorname{alg} Z$ & XOO4009 & $\begin{array}{l}\text { two-component system } \\
\text { sensor protein }\end{array}$ & 13.28 & 19.44 & 0.44 & 0.38875 & FALSE \\
\hline- & XOO4201 & $\begin{array}{l}\text { two-component system } \\
\text { sensor protein }\end{array}$ & 20.73 & 26.41 & 0.24 & 0.57069 & FALSE \\
\hline$n t r C$ & XOO4202 & $\begin{array}{l}\text { two-component system } \\
\text { regulatory protein }\end{array}$ & 8.72 & 15.56 & 0.72 & 0.22512 & FALSE \\
\hline smeR & XOO4341 & $\begin{array}{l}\text { two-component system } \\
\text { regulatory protein }\end{array}$ & 7.69 & 18.97 & 1.19 & 0.04211 & FALSE \\
\hline
\end{tabular}


Table 2. Cont.

\begin{tabular}{|c|c|c|c|c|c|c|c|}
\hline \multirow{2}{*}{ Gene } & \multirow{2}{*}{ Locus Tag } & \multirow{2}{*}{ Product } & \multicolumn{2}{|c|}{ RPKM } & \multicolumn{3}{|c|}{ DEGseq Analysis } \\
\hline & & & 10331 & 1009 & $\log 2$ FC & $p$-Value & Signature * \\
\hline$n t r C$ & XOO4483 & $\begin{array}{l}\text { two-component system } \\
\text { regulatory protein }\end{array}$ & 13.97 & 20.70 & 0.46 & 0.35612 & FALSE \\
\hline$n \operatorname{tr} B$ & XOO4484 & $\begin{array}{l}\text { two-component system } \\
\text { sensor protein }\end{array}$ & 40.25 & 58.94 & 0.44 & 0.13231 & FALSE \\
\hline $\operatorname{tcs} R$ & XOO4543 & $\begin{array}{l}\text { two-component system } \\
\text { regulatory protein }\end{array}$ & 7.25 & 18.38 & 1.23 & 0.04017 & FALSE \\
\hline $\operatorname{raxP}$ & XOO3397 & $\begin{array}{c}\text { sulfate adenylyltransferase } \\
\text { subunit } 2\end{array}$ & 10.46 & 18.71 & 0.73 & 0.18138 & FALSE \\
\hline $\operatorname{colS}(\operatorname{raxH})$ & XOO3534 & $\begin{array}{l}\text { two-component system } \\
\text { sensor protein }\end{array}$ & 7.85 & 14.60 & 0.78 & 0.20791 & FALSE \\
\hline $\operatorname{cols}(\operatorname{raxR})$ & XOO3535 & $\begin{array}{l}\text { two-component system } \\
\text { regulatory protein }\end{array}$ & 11.88 & 18.41 & 0.52 & 0.32541 & FALSE \\
\hline $\operatorname{rax} B$ & XOO3543 & $\begin{array}{l}\mathrm{ABC} \text { transporter } \\
\text { protein RaxB }\end{array}$ & 8.04 & 15.86 & 0.87 & 0.15188 & FALSE \\
\hline $\operatorname{rax} A$ & XOO3544 & $\begin{array}{l}\text { membrane fusion } \\
\text { protein RaxA }\end{array}$ & 3.86 & 12.99 & 1.64 & 0.03244 & FALSE \\
\hline $\operatorname{rax} S T$ & XOO3546 & sulfotransferase RaxST & 11.06 & 12.49 & 0.07 & 0.91282 & FALSE \\
\hline
\end{tabular}

Quorum sensing (QS) is the process in which bacteria monitor their own population density by sensing the levels of extracellular signal molecules that are released by the microorganisms, and the QS systems might enable pathogens to overcome the host defense mechanisms [33]. RpfC and RpfG serve as a two-component system for the perception and transduction of the extracellular diffusible signal factor (DSF) family signal-mediated QS [34-36]. The expression pattern of the RpfC/RpfG two-component regulatory system (XOO2870 and XOO2871) associated with QS was different between the HB1009 and KACC10331 strains (Table S1). Furthermore, Ryan et al. [37] reported that several domain proteins, including GGDEF, EAL, and HD-GYP, are involved in a network of signal transduction systems for responding to different environmental factors to modulate the level of the second messenger cyclic di-GMP in X. campestris pv. campestris. In this study, a TCS regulatory protein with a GGDEF (XOO2787) or with both GGDEF and EAL domains (XOO0520) had upregulated ( 0.59 and $1.26 \log 2 \mathrm{FC}$, respectively) expression in the HB1009 strain. In contrast, a TCS regulatory protein with an HD-GYP domain (XOO2798) had significantly downregulated expression $(-2.2 \log 2$ FC) in the HB1009 strain compared with the KACC10331 strain. In addition, XOO2860 (encoding cyclic di-GMP phosphodiesterase A) had upregulated expression in the HB1009 strain compared with the KACC10331 strain (Table S2).

Rice lines carrying Xa21 (encodes a leucine-rich repeat receptor-like kinase) are able to induce an effective defense response to multiple strains of the bacterial Xoo pathogens [38]. In addition, several genes that are required for activation of XA21-mediated immunity ( $r a x)$ have been identified in Xoo [13-15]. Furthermore, Lee et al. [39] and Zhang et al. [31] reported that Xoo requires a regulatory TCS called RaxRH to regulate expression of $10 \operatorname{rax}$ genes, including $\operatorname{rax} A, \operatorname{rax} B, \operatorname{rax} C, \operatorname{rax} S T, \operatorname{rax} P, \operatorname{rax} Q$, $\operatorname{raxR}, \operatorname{raxR} 2, \operatorname{raxH}$, and $\operatorname{raxH2}$. Our results revealed that all of these $\operatorname{rax}$ genes were not expressed at significantly different levels between the HB1009 and KACC10331 strains according to the DEGseq analysis ( $p$-value $>0.001$, FALSE), except for raxR2 (XOO0423) (Table 2, Table S2). However, some of these genes, including $\operatorname{raxP}$ (XOO3397), $\operatorname{raxR}$ (XOO3535), $\operatorname{raxB}$ (XOO3543), $\operatorname{raxA}$ (XOO3544), and raxST (XOO3546), showed different expression patterns between the two strains in the qRT-PCR analysis (Figure 5). As described above, the expression of $\operatorname{raxR2}$ (XOO0423) associated with TCSs was significantly downregulated in the HB1009 (K3a race) strain (Table 2, Table S1), which showed reduced virulence against IRBB21 (carrying Xa-21) (Figure 1). In contrast, $\operatorname{raxR}$ (XOO3535) was upregulated in the HB1009 (K3a race) strain, although the raxR gene did not show significantly different expression levels in the DEGseq analysis ( $p$-value > 0.001, FALSE) (Table 2, Table S2). The findings of the current study are consistent with those of Lee et al. [39] who reported that a response regulator encoded by phoP $(\operatorname{raxR} 2 ; \mathrm{XOO} 0423)$ is upregulated in a $\operatorname{raxR}$ gene knockout mutant strain. The PhoPQ-regulated protein 
(XOO1731) not only is required for AvrXa21 activity but also regulates various cellular activities as a regulator of virulence in Salmonella and other species [40-42]. Upregulation of the phoPQ-regulated protein was detected in the HB1009 strain, although this gene did not show significantly different expression levels in the DEGseq analysis ( $p$-value $>0.001$, FALSE).

\section{Discussion}

In the past several years, a large number of Xoo pathogenesis-related genes have been isolated and characterized, as many researchers have tried to elucidate the mechanisms of the Xoo-rice interaction. However, many aspects of the mechanisms of the Xoo-rice interaction are still not clearly understood. For example, although several genes involved in the activation of XA21-mediated immunity have been identified, a key gene required to elicit rice-resistant protein Xa21 expression has not been identified [43]. As described earlier, control of BB involves the introduction of resistance genes to confer major gene-specific resistance against some pathogen races. However, this type of resistance frequently results in rapid changes in the pathogen diversity, and new races of the pathogen are able to overcome the deployed resistance [44,45].

In this study, we presented the comparison of genome-wide transcriptional patterns between two Xoo strains and used transcriptome profiling to identify genes that might be involved in the different pathotypes and that may be specific to the Xoo race. GO term enrichment analysis identified four remarkably enriched GO terms in the upregulated genes, and the expression of 44 genes (a total of 147 enriched genes) was considerably higher (log2 fold changes $>2$ ) in the HB1009 (K3a race) strain than in the Xoo KACC10331 (K1 race) strain (Table S3). Among the 44 genes, 13 and seven genes were identified as transposase and hypothetical genes, respectively. In addition, six genes, identified as transcription regulators, showed a higher level of expression in the HB1009 (K3a race) strain than in the Xoo KACC10331 (K1 race) strain. Although the additional research is needed to determine the biological function of these genes, these results suggest that these enriched genes might be involved in host-specific immunity responses or facilitate virulence processes in the pathogen. Xoo virulence and the regulatory genes required for pathogenicity are commonly found in pathogenicity islands (PAIs) that encode for a type III secretion system (TTSS) assembled from hrp gene products [46-48]. In this study, several genes associated with the expression of $h r p$ genes were significantly upregulated in HB1009. This result suggests that the expression levels of these hrp genes may differ for these two strains in rice varieties, since $h r p$ genes were shown to be essential for bacterial pathogenicity in susceptible hosts and hypersensitive reaction (HR) induction in host and non-host plants in the plant pathogen $[47,49]$. TAL effectors also play key roles in host immune responses or virulence processes in the pathogen, and the genomic sequences revealed that Philippine (PXO99A), Japanese (MAFF311018), and Korean (KACC10331) strains contain 19, 17, and 15 TAL effector genes, respectively. In addition, Southern hybridization analysis revealed that the HB1009 strain contains 13 TAL effector genes in its chromosome (data not shown). Due to the repetitive structure of the TAL effector coding sequences, NGS-based transcriptome analysis is not sufficient to evaluate their expression. Although additional approaches such as site-directed mutagenesis of these TAL effectors and evaluation of host immunity responses are needed to facilitate the understanding of the function of these TAL effectors, transcriptome analysis revealed that the expression levels of these genes were slightly upregulated in the HB1009 strain; however, these genes did not show significantly different expression levels in the DEGseq analysis ( $p$-value $>0.001$, FALSE).

Zhang et al. [31] reported that the bacterial motilities were significantly different among the Xoo strains (C5, China race 5; P2 and P6, Philippines race 2 and 6, respectively), which showed different virulence patterns against various rice cultivars. This study also demonstrated that the genes encoding Hrp proteins and $\mathrm{T} 3$ effectors were significantly downregulated in $\mathrm{C} 5$, whereas no significantly different virulence pattern was observed among those strains in a virulence assay with various rice cultivars. Thus, they hypothesized that strong motility might compensate for the weaker expression of Hrp proteins in C5, which allows C5 to exhibit similar virulence levels with the other two Xoo strains. 
We also conducted a bacterial motility assay with the KACC10331 and HB1009 strains on semi-solid swarm plates. However, there was no significant difference in bacterial motility between the two strains (data not shown). These results suggest that the different virulence patterns of the two Xoo strains might not be due to differences in bacterial motility but, rather, might be due to differently expressed genes, including hrp and TCSs, as well as an enriched gene set according to the enrichment analysis between the two Xoo strains.

Although further studies are required to elucidate the relationship between the differently expressed genes and the Xoo-rice interaction, our findings should facilitate the identification of genes that are involved in this interaction, especially elicitors for the expression of the rice-resistance genes such as avrXa21, and will provide valuable insights to aid in developing future strategies to control BB caused by newly evolved strains such as HB1009 (K3a).

\section{Materials and Methods}

\subsection{Bacterial Strains and Culture Conditions}

The Xoo KACC10331 (K1 race) and HB1009 (K3a race) strains were obtained from the National Institute of Crop Science in Jeonju, Korea, and were cultured on YDC medium (2.0\% D-glucose, 2.0\% $\mathrm{CaCO}_{3}, 1.0 \%$ yeast extract, and $1.5 \%$ agar) or in nutrient broth (3\% beef extract, $5 \%$ peptone, and $1.5 \%$ agar; Difco) at $28^{\circ} \mathrm{C}$ until $2.0 \mathrm{OD}_{600}$. For total RNA isolation, the Xoo strains were washed twice and immediately transferred into XOM2 medium $(0.18 \%$ xylose sugar, $670 \mathrm{mM}$ D,L-methionine, $10 \mathrm{mM}$ sodium l(+)-glutamate, $14.7 \mathrm{mM} \mathrm{KH}_{2} \mathrm{PO}_{4}, 40 \mathrm{mM} \mathrm{MnSO}_{4}, 240 \mathrm{mM} \mathrm{Fe}(\mathrm{III}) \mathrm{EDTA}$, and $5 \mathrm{mM} \mathrm{MgCl}_{2}$, $\mathrm{pH}$ 6.5).

\subsection{Virulence Assay}

Seeds of the rice varieties were sown in a seedling nursery, and 30-day-old seedlings were transplanted. Leaves of 50-day-old (at the tillering stage) rice varieties were artificially infected with the Xoo strains $\left(10^{9}\right.$ cells $\left.\cdot \mathrm{mL}^{-1}\right)$ by clipping the leaf tips with sterile scissors. The infected rice plants were then grown and maintained under greenhouse conditions $\left(25-30{ }^{\circ} \mathrm{C}, 60 \%\right.$ relative humidity). Distilled water was used as the control treatment. Twenty-one days after inoculation, the means values and standard deviations (SDs) of lesion lengths were calculated for each triplicate set of experiments.

\subsection{Total RNA Isolation, Illumina Sequencing, and Data Analysis}

Total RNA was extracted from a stationary phase culture $\left(\mathrm{OD}_{600 \mathrm{~nm}} 0.8\right)$ using an RNeasy mini kit according to the manufacturer's instructions (Qiagen, Hilden, Germany). Total RNA was further treated using RNase-free DNase set (Qiagen, Hilden, Germany) following manufacturer's instructions to discard DNA contamination. Next-generation sequencing (NGS) library preparation and sequencing were performed with $1 \mu \mathrm{g}$ of each total RNA using the Illumina HiSeq2000 platform in accordance with the manufacturer's protocol (Illumina, Inc., San Diego, CA, USA). The raw data were deposited to the National Center for Biotechnology Information (NCBI) Sequence Reads Archive (SRA) with accession numbers SRP066131 (Xoo KACC10331) and SRP066133 (Xoo HB1009). Sequencing reads were processed by SolexaQA software [50] to control the quality of raw data. The trimmed reads were mapped to the Xoo KACC 10331 genome (NCBI accession no. NC_006834) using Bowtie aligner (http://bowtie-bio.sourceforge.net/index.shtml). The expression level was quantified by the reads per kilobase per million mapped reads (RPKM). DEGseq [25] was applied to identify differentially expressed genes (DEGs) with significance defined as a $p$-value less than 0.001. Gene Ontology (GO) term annotation and enrichment analysis of the upregulated and downregulated genes were carried out using Blast2GO software [26]. 


\subsection{Quantitative Real-Time RT-PCR Assay}

To validate the RNA-seq data, a subset of differentially expressed genes (DEGs) was verified by quantitative real-time RT-PCR (qRT-PCR). An independent set of cell cultures and total RNA samples from the two Xoo strains were prepared following the same protocol as for the Illumina analysis. The sequence of each gene was obtained from the Xoo 10331 database (http://www.ncbi.nlm.nih.gov) and used for designing primers by IDT (Integrated DNA Technologies, Coralville, IA, USA, https://eu.idtdna.com/Primerquest/Home/Index). RNA samples from three independent replicates were treated with DNase before cDNA synthesis. Quantitative real-time PCR analysis was performed using a RotorGene 6000 system (Qiagen, Hilden, Germany) in $25-\mu \mathrm{L}$ reactions containing $12.5 \mu \mathrm{L}$ SensiFAST SYBR No-ROX kit (Bioline, Sydney, Australia), 5 pmol of each primer (Table S5), and $25 \mathrm{ng}$ of cDNA template. Reaction conditions were as follows: $3 \mathrm{~min}$ of holding at $95^{\circ} \mathrm{C}$ followed by 40 cycles of $95^{\circ} \mathrm{C}$ for $5 \mathrm{~s}, 60{ }^{\circ} \mathrm{C}$ for $10 \mathrm{~s}$, and $72{ }^{\circ} \mathrm{C}$ for $15 \mathrm{~s}$. The gene expression levels (arbitrary units) were normalized on the basis of transcript amounts of 16S RNA as an internal reference. The relative expression level of each gene is defined as $\Delta C \mathrm{t}=C \mathrm{t}_{\text {target }}-\mathrm{Ct}_{16 \mathrm{~S}}$ to represent the difference between the transcript abundance of genes examined and the transcript abundance of 16S RNA.

Supplementary Materials: Supplementary materials can be found at http://www.mdpi.com/1422-0067/ $17 / 2 / 259 /$ s1.

Acknowledgments: This study was supported by a grant to the Agenda program (Project No. PJ0086812014) of the National Institute of Crop Science, Rural Development Administration, Republic of Korea.

Author Contributions: Young-Jin Park and Tae-Hwan Noh designed the experiments. Young-Jin Park, Eun-Sung Song, and Hong-Il Kim performed the experiments and analyzed the data. Tae-Hwan Noh and Mi-Hyung Kang contributed reagents and materials. Young-Jin Park wrote and edited the paper. All authors reviewed the manuscript.

Conflicts of Interest: The authors declare no conflict of interest. The founding sponsors had no role in the design of the study; in the collection, analyses, or interpretation of data; in the writing of the manuscript; and in the decision to publish the results.

$\begin{array}{ll}\begin{array}{l}\text { Abbreviations } \\ \text { Xoo }\end{array} & \begin{array}{l}\text { Xanthomonasoryzae pv. oryzae } \\ \text { bacterial blight } \\ \text { BB }\end{array} \\ \text { hypersensitive response and pathogenicity } \\ \text { TCSs } & \text { two-component regulatory systems } \\ \text { PRRs } & \text { pattern recognition receptors } \\ \text { QS } & \text { quorum sensing } \\ \text { DSF } & \text { diffusible signal factor } \\ \text { rax } & \text { required for activation of XA21-mediated immunity } \\ \text { TTSS } & \text { type III secretion system } \\ \text { DEGs } & \text { differentially expressed genes } \\ \text { qRT-PCR } & \text { quantitative real-time RT-PCR }\end{array}$

\section{References}

1. Mew, T.W. Current status and future prospects of research on bacterial blight of rice. Annu. Rev. Phytopathol. 1987, 25, 359-382. [CrossRef]

2. Bhasin, H.; Bhatia, D.; Raghuvanshi, S.; Lore, J.S.; Sahi, G.K.; Kaur, B.; Vikal, Y.; Singh, K. New PCR-based sequence-tagged site marker for bacterial blight resistance gene Xa38 of rice. Mol. Breed. 2012, 30, 607-611. [CrossRef]

3. Natrajkumar, P.; Sujatha, K.; Laha, G.S.; Srinivasarao, K.; Mishra, B.; Viraktamath, B.C.; Hari, Y.; Reddy, C.S.; Balachandran, S.M.; Ram, T.; et al. Identification and fine-mapping of Xa33, a novel gene for resistance to Xanthomonas oryzae pv. oryzae. Phytopathology 2012, 102, 222-228. 
4. Song, W.Y.; Pi, L.Y.; Wang, G.L.; Gardner, J.; Holstion, T.; Ronald, P.C. Evolution of the rice Xa21 disease resistance gene family. Plant Cell 1997, 9, 1279-1287. [CrossRef] [PubMed]

5. Yang, D.; Sanchez, A.; Khush, G.S.; Zhu, Y.; Huang, N. Construction of a BAC contig containing the $x a 5$ locus in rice. Theor. Appl. Genet. 1998, 97, 1120-1124. [CrossRef]

6. Sun, X.; Yang, Z.; Wang, S.; Zhang, Q. Identification of a $47 \mathrm{~kb}$ DNA fragment containing Xa4, a locus for bacterial blight resistance in rice. Theor. Appl. Genet. 2003, 106, 683-687. [PubMed]

7. Gu, K.; Yang, B.; Tian, D.; Wu, L.; Wang, D.; Sreekala, C.; Yang, F.; Chu, Z.; Wang, G.L.; White, F.F.; et al. $R$ gene expression induced by a type-III effector triggers disease resistance in rice. Nature 2005, 435, 1122-1125. [CrossRef] [PubMed]

8. Niño-Liu, D.O.; Ronald, P.C.; Bogdanove, A.J. Xanthomonas oryzae pathovars: Model pathogens of a model crop. Mol. Plant Pathol. 2006, 7, 303-324. [CrossRef] [PubMed]

9. Cheema, K.; Grewal, N.; Vikal, Y.; Sharma, R.; Lore, J.S.; Das, A.; Bhatia, D.; Mahajan, R.; Gupta, V.; Bharaj, T.S.; et al. A novel bacterial blight resistance gene from Oryza nivara mapped to $38 \mathrm{~kb}$ region on chromosome 4L and transferred to Oryza sativa L. Genet. Res. 2008, 90, 397-407. [CrossRef] [PubMed]

10. Tian, D.; Wang, J.; Zeng, X.; Gu, K.; Qiu, C.; Yang, X.; Zhou, Z.; Goh, M.; Luo, Y.; Murata-Hori, M.; et al. The rice TAL effector-dependent resistance protein XA10 triggers cell death and calcium depletion in the endoplasmic reticulum. Plant Cell 2014, 26, 497-515. [CrossRef] [PubMed]

11. Wang, C.; Zhang, X.; Fan, Y.; Gao, Y.; Zhu, Q.; Zheng, C.; Qin, T.; Li, Y.; Che, J.; Zhang, M.; et al. XA23 is an executor $\mathrm{R}$ protein and confers broad-spectrum disease resistance in rice. Mol. Plant 2015, 8, 290-302. [CrossRef] [PubMed]

12. Khush, G.S.; Bacalangco, E.; Ogawa, T. A new gene for resistance to bacterial blight from O. longistaminata. Rice Genet. Newsl. 1990, 7, 121-122.

13. Shen, Y.; Sharma, P.; da Silva, F.G.; Ronald, P. The Xanthomonas oryzae pv. oryzae raxP and $\operatorname{rax} Q$ genes encode an ATP sulfurylase and adenosine- $5^{\prime}$-phosphosulphate kinase that are required for AvrXa21 avirulence activity. Mol. Microbiol. 2002, 44, 37-48. [PubMed]

14. Burdman, S.; Shen, Y.; Lee, S.W.; Xue, Q.; Ronald, P.C. RaxH/RaxR: A two-component regulatory system in Xanthomonas oryzae pv. oryzae required for AvrXa21 activity. Mol. Plant Microbe Interact. 2004, 17, 602-612. [CrossRef] [PubMed]

15. Da Silva, F.G.; Shen, Y.; Dardick, C.; Burdman, S.; Yadav, R.C.; de Leon, A.L.; Ronald, P.C. Bacterial genes involved in type I secretion and sulfation are required to elicit the rice Xa21-mediated innate immune response. Mol. Plant Microbe Interact. 2004, 17, 593-601. [CrossRef] [PubMed]

16. Lee, S.W.; Han, S.W.; Sririyanum, M.; Park, C.J.; Seo, Y.S.; Ronald, P.C. A type I-secreted, sulfated peptide triggers XA21-mediated innate immunity. Science 2009, 326, 850-853. [CrossRef] [PubMed]

17. Lee, S.W.; Han, S.W.; Sririyanum, M.; Park, C.J.; Seo, Y.S.; Ronald, P.C. Retraction. A type I-secreted, sulfated peptide triggers XA21-mediated innate immunity. Science 2013, 342, 191. [CrossRef] [PubMed]

18. Yun, M.S.; Lee, E.J.; Cho, Y.S. Pathogenic specialization of the rice bacterial leaf blight pathogen, Xanthomonas campestris pv. oryzae: Race classification based on reactions of Korean differential varieties. Korean J. Appl. Entomol. 1985, 24, 97-101.

19. Shin, M.S.; Shin, H.T.; Jun, B.T.; Choi, B.S. Effect of inoculation of compatible and incompatible bacterial blight races on grain yield and quality of two rice cultivars. Korean J. Breed. Sci. 1992, 24, 264-267.

20. Noh, T.H.; Lee, D.K.; Park, J.C.; Shim, H.K.; Choi, M.Y.; Kang, M.H.; Kim, J.D. Effect of bacterial leaf blight occurrence on rice yield and grain quality in different rice growth stage. Res. Plant Dis. 2007, 13, $20-23$. [CrossRef]

21. Kim, K.Y.; Shin, M.S.; Kim, W.J.; Mo, Y.J.; Nam, J.K.; Noh, T.H.; Kim, B.K.; Ko, J.K. Effective combination of resistance genes against rice bacterial blight pathogen. Korean J. Breed. Sci. 2009, 41, 244-251.

22. Noh, T.H.; Lee, D.K.; Kang, M.H.; Shin, M.S.; Na, S.Y. Identification of new race of Xanthomonas oryzae pv. oryzae (Xoo) in Korea. Phytopathology 2003, 93, S66.

23. Song, E.S.; Kim, S.Y.; Noh, T.H.; Cho, H.; Chae, S.C.; Lee, B.M. PCR-based assay for rapid and specific detection of the new Xanthomonas oryzae pv. oryzae K3a race using an AFLP-derived marker. J. Microbiol. Biotechnol. 2014, 24, 732-739. [PubMed]

24. Lee, B.M.; Park, Y.J.; Park, D.S.; Kang, H.W.; Kim, J.G.; Song, E.S.; Park, I.C.; Yoon, U.H.; Hahn, J.H.; Koo, B.S.; et al. The genome sequence of Xanthomonas oryzae pathovar oryzae KACC10331, the bacterial blight pathogen of rice. Nucleic Acids Res. 2005, 33, 577-586. [CrossRef] [PubMed] 
25. Wang, L.; Feng, Z.; Wang, X.; Wang, X.; Zhang, X. DEGseq: An R package for identifying differentially expressed genes from RNA-seq data. Bioinformatics 2010, 26, 136-138. [CrossRef] [PubMed]

26. Conesa, A.; Götz, S.; García-Gómez, J.M.; Terol, J.; Talón, M.; Robles, M. Blast2GO: A universal tool for annotation, visualization and analysis in functional genomics research. Bioinformatics 2005, 21, 3674-3676. [CrossRef] [PubMed]

27. Kim, J.G.; Park, B.K.; Yoo, C.H.; Jeon, E.; Oh, J.; Hwang, I. Characterization of the Xanthomonas axonopodis pv. glycines Hrp pathogenicity island. J. Bacteriol. 2003, 185, 3155-3166. [PubMed]

28. Cho, H.J.; Park, Y.J.; Noh, T.H.; Kim, Y.T.; Kim, J.G.; Song, E.S.; Lee, D.H.; Lee, B.M. Molecular analysis of the hrp gene cluster in Xanthomonas oryzae pathovar oryzae KACC10859. Microb. Pathog. 2008, 44, 473-483. [CrossRef] [PubMed]

29. Wengelnik, K.; Rossier, O.; Bonas, U. Mutations in the regulatory gene hrpG of Xanthomonas campestris pv. vesicatoria result in constitutive expression of all hrp genes. J. Bacteriol. 1999, 181, 6828-6831. [PubMed]

30. Wengelnik, K.; Bonas, U. HrpXv, an AraC-type regulator, activates expression of five of the six loci in the $h r p$ cluster of Xanthomonas campestris pv. vesicatoria. J. Bacteriol. 1996, 178, 3462-3469. [PubMed]

31. Zhang, F.; Du, Z.; Huang, L.; Cruz, C.V.; Zhou, Y.; Li, Z. Comparative transcriptome profiling reveals different expression patterns in Xanthomonas oryzae pv. oryzae strains with putative virulence-relevant genes. PLOS ONE 2013, 8, e64267.

32. Charles, T.C.; Jin, S.; Nester, E.W. Two-component sensory transduction systems in phytobacteria. Annu. Rev. Phytopathol. 1992, 30, 463-484. [CrossRef] [PubMed]

33. Ng, W.L.; Bassler, B.L. Bacterial quorum-sensing network architectures. Annu. Rev. Genet. 2009, 43, $197-222$. [CrossRef] [PubMed]

34. Slater, H.; Alvarez-Morales, A.; Barber, C.E.; Daniels, M.J.; Dow, J.M. A two component system involving an HD-GYP domain protein links cell-cell signaling to pathogenicity gene expression in Xanthomonas campestris. Mol. Microbiol. 2000, 38, 986-1003. [CrossRef] [PubMed]

35. Ryan, R.P.; Fouhy, Y.; Lucey, J.F.; Crossman, L.C.; Spiro, S.; He, Y.W.; Zhang, L.H.; Heeb, S.; Camara, M.; Williams, P.; et al. Cell-cell signaling in Xanthomonas campestris involves an HD-GYP domain protein that functions in cyclic di-GMP turnover. Proc. Natl. Acad. Sci. USA 2006, 103, 6712-6717. [CrossRef] [PubMed]

36. Guo, Y.; Zhang, Y.; Li, J.L.; Wang, N. Diffusible signal factor-mediated quorum sensing plays a central role in coordinating gene expression of Xanthomonas citri subsp. citri. Mol. Plant Microbe Interact. 2012, 25, 165-179. [CrossRef] [PubMed]

37. Ryan, R.P.; McCarthy, Y.; Andrade, M.; Farah, C.S.; Armitage, J.P.; Dow, J.M. Cell-cell signal-dependent dynamic interactions between HD-GYP and GGDEF domain proteins mediate virulence in Xanthomonas campestris. Proc. Natl. Acad. Sci. USA 2010, 107, 5989-5994. [CrossRef] [PubMed]

38. Song, W.Y.; Wang, G.L.; Chen, L.L.; Kim, H.S.; Pi, L.Y.; Holsten, T.; Gardner, J.; Wang, B.; Zhai, W.X.; Zhu, L.H.; et al. A receptor kinase-like protein encoded by the rice disease resistance gene, Xa21. Science 1995, 270, 1804-1806. [CrossRef] [PubMed]

39. Lee, S.W.; Han, S.W.; Bartley, L.E.; Ronald, P.C. Unique characteristics of Xanthomonas oryzae pv. oryzae AvrXa21 and implications for plant innate immunity. Proc. Natl. Acad. Sci. USA 2006, 103, 18395-18400. [CrossRef] [PubMed]

40. Zwir, I.; Shin, D.; Kato, A.; Nishino, K.; Latifi, T.; Solomon, F.; Hare, J.M.; Huang, H.; Groisman, E.A. Dissecting the PhoP regulatory network of Escherchia coli and Salmonella enterica. Proc. Natl. Acad. Sci. USA 2005, 102, 2862-2867. [CrossRef] [PubMed]

41. Garcia-Calderon, C.B.; Casadesús, J.; Ramos-Morales, F. Rcs and PhoPQ regulatory overlap in the control of Salmonella enterica virulence. J. Bacteriol. 2007, 189, 6635-6644. [CrossRef] [PubMed]

42. Lee, S.W.; Jeong, K.S.; Han, S.W.; Lee, S.E.; Phee, B.K.; Hahn, T.R.; Ronald, P. The Xanthomonas oryzae pv. oryzae PhoPQ two-component system is required for AvrXA21 activity, hrpG expression, and virulence. J. Bacteriol. 2008, 190, 2183-2197. [PubMed]

43. Bahar, O.; Pruitt, R.; Luu, D.D.; Schwessinger, B.; Daudi, A.; Liu, F.; Ruan, R.; Fontaine-Bodin, L.; Koebnik, R.; Ronald, P. The Xanthomonas Ax21 protein is processed by the general secretory system and is secreted in association with outer membrane vesicles. Peer J. 2014, 2, e242. [CrossRef] [PubMed]

44. Leach, J.E.; White, F.F. Bacterial avirulence genes. Annu. Rev. Phytopathol. 1996, 34, 153-179. [CrossRef] [PubMed] 
45. Leach, J.E.; Davidson, R.; Liu, B.; Manosalva, P.; Mauleon, R.; Carrillo, G.; Bruce, M.; Stephens, J.; Diaz, M.G.; Nelson, R.; et al. Understanding broad-spectrum durable resistance in rice. In Rice Genetics V; Brar, D.S., Mackill, D.J., Hardy, B., Eds.; World Scientific: Toh Tuck, Singapore, 2007; pp. 191-207.

46. Pugsley, A.P. The complete general secretory pathway in gram-negative bacteria. Microbiol. Rev. 1993, 57, 50-108. [PubMed]

47. Lindgren, P.B. The role of hrp genes during plant-bacterial interactions. Annu. Rev. Phytopathol. 1997, 35, 129-152. [CrossRef] [PubMed]

48. He, S.Y. Type III protein secretion systems in plant and animal pathogenic bacteria. Annu. Rev. Phytopathol. 1998, 36, 363-392. [CrossRef] [PubMed]

49. Lindgren, P.B.; Peet, R.C.; Panopoulos, N.J. Gene-cluster of Pseudomonas syringae pv. "phaseolicola" controls pathogenicity of bean plants and hypersensitivity of nonhost plants. J. Bacteriol. 1986, 168, 512-522. [PubMed]

50. Cox, M.P.; Peterson, D.A.; Biggs, P.J. SolexaQA: At a glance quality assessment of illumine second-generation sequencing data. BMC Bioinform. 2010. [CrossRef] [PubMed]

(C) 2016 by the authors; licensee MDPI, Basel, Switzerland. This article is an open access article distributed under the terms and conditions of the Creative Commons by Attribution (CC-BY) license (http://creativecommons.org/licenses/by/4.0/). 